\title{
Predictors of disengagement from Cognitive Behavioural Therapy for psychosis in a National Health Service setting: A retrospective evaluation
}

\begin{abstract}
Objectives: To evaluate whether demographic and clinical variables are related to disengagement rates in Cognitive Behavioural Therapy (CBT) for psychosis in a clinical setting.
\end{abstract}

Methods: The medical records and symptom severity data (from Health of the Nation Outcome Scales (HoNOS)) were analysed retrospectively for 103 referrals for CBT for psychosis in National Health Service (NHS) secondary care and Early Intervention in Psychosis teams.

Results: Overall 42.7\% ( $n=44)$ disengaged from CBT. There was no impact of gender or ethnicity, and no impact of clinical variables such as risk history and comorbid diagnosis. However risk of disengagement was significantly higher for those who were younger, $F=6.89$, partial $\eta^{2}=.064, p=<.05$; had greater total HoNOS scores, $F=4.22$, partial $\eta^{2}=.04$, $p<.05$; and more severe symptoms on the HoNOS items of overactive, aggressive, disruptive or agitated behaviour, $\chi^{2}=6.13, p<.01$; problem drinking or drug taking, $\chi^{2}=7.65, p<.05$; depressed mood, $\chi^{2}=7.0, p<.01$; and problems with occupation and activities: $\chi^{2}=3.68, p<.05$. There was a non-significant trend for shorter waiting times to be associated with greater levels of disengagement.

Conclusions: These results indicate that it may not be psychosis per se that disrupts engagement in CBT, but linked behavioural and emotional factors. A more assertive approach to these factors - overactive, aggressive, disruptive or agitated behaviour, problem drinking or drug taking, and depressed mood, and problems with occupation and activities, particularly in younger people - may be valuable prior to or early on in therapy as a means of increasing engagement in CBT for psychosis. 
Declaration of Interest: None to declare.

Keywords: Psychosis; Psychotic; Schizophrenia; CBT; Therapy; Disengagement. 


\section{Practitioner Points}

- Risk of disengagement from CBT for psychosis increases with overactive, aggressive, disruptive or agitated behaviour (54.9\% vs. $30.8 \%$ ), problem drinking and drug taking (61.1\% vs. $32.8 \%)$, depressed mood (56\% vs. $30.2 \%)$, and problems with occupation and activities (53.3\% vs. 34.5\%), with a trend for younger age.

- An assertive and motivational approach to engagement and a focus on addressing low mood and problematic behaviours, prior to or early in therapy may be warranted, particularly for younger people.

- This evaluation is limited by small sample size and being retrospective.

- These results speak to the question of whether psychosis itself renders people inappropriate for CBT for psychosis, or whether problems arise due to behavioural and emotional factors that might be addressed to increase access to CBT for psychosis. 


\section{Predictors of disengagement from Cognitive Behavioural Therapy for psychosis in a National Health Service setting: A retrospective evaluation}

\section{Introduction}

Cognitive Behavioural Therapy (CBT) is recommended for people with psychosis (NICE, 2014). However, high levels of disengagement from multi-disciplinary services (Doyle et al., 2014; Kreyenbuhl, Nossel, \& Dixon, 2009) raise questions about engagement rates for CBT for psychosis.

Doyle et al. (2014) sought to explain disengagement rates of around 30\% from First Episode Psychosis (FEP) services, and found that substance misuse and lack of involvement of a family member were consistent predictors of disengagement across studies. These authors found conflicting results for symptom severity and duration of untreated psychosis, and limited or no evidence for impact of demographic variables - age, ethnicity, gender and marital status. Kreyenbuhl et al. (2009) found similar rates of disengagement for people with a diagnosis of schizophrenia from a range of services, and that this was more likely in those who were male, younger, from an ethnic minority, had poor social functioning, early onset and comorbid mental health or substance use problems.

Importantly, there is very little research examining predictors of disengagement from psychological therapies for psychosis. Fanning et al. (2012) investigated disengagement from group CBT for FEP, finding that those with higher educational levels and fewer negative symptoms were more likely to complete therapy. A service evaluation of an Increasing Access for Psychological Therapies (IAPT) pilot site for CBT for psychosis found no differences in therapy completion based on gender or ethnicity (Jolley et al., 2015).

This evaluation aimed to examine predictors of disengagement from CBT for psychosis in two National Health Service (NHS) teams. Given the modest and mixed 
literature regarding therapy, demographic and clinical variables to date, and the routinely collected data available, we explored the following evaluation questions:

(i) What is the impact of therapy variables (waiting time; whether low or high intensity CBT) on disengagement?

(ii) What is the impact of demographic variables (gender; age; ethnicity) on disengagement?

(iii) What is the impact of clinical variables (diagnosis; comorbid diagnosis; EIP / CMHT; positive psychotic symptoms at last nurse or psychiatrist assessment before referral; acute service input in 12 months prior to referral; history of physical aggression to others; history of self-harm or suicidal behaviour; history of substance misuse) on disengagement?

(iv) What is the impact of current problem severity on disengagement?

\section{Methods}

\section{Design}

The evaluation used a case-controlled design via a retrospective examination of existing data. The dependent variable was disengagement from CBT. The predictor variables were:

- Therapy variables: Time spent waiting for therapy, Low Intensity (LI) vs. HighIntensity (HI) CBT.

- Demographic variables: Gender, age, ethnicity.

- Clinical variables: Diagnosis, comorbid diagnosis, EIP / CMHT, positive psychotic symptoms at last nurse or psychiatrist assessment before referral, acute service input in the 12 months prior to referral, history of physical aggression to others, history of selfharm or suicidal behaviour, historical substance misuse.

- Health of the National Outcome Scales (HoNOS, Wing et al., 1998): This is a 12-item clinician-rated assessment of current complexity / severity of mental health problems. Example items include "problems associated with hallucinations and delusions" and 
"problem drinking and drug taking." Items are rated from 0-4 ("no problem," "minor problem," "mild problem," "moderately severe problem" and "severe to very severe problem"). Amin et al. (1999) demonstrated concurrent and inter-rater reliability of the HoNOS for people with FEP. The measure had acceptable reliability in the current sample: $\alpha=.75$.

\section{Participants}

We examined data from referrals for CBT for psychosis via an NHS Community Mental Health Team (CMHT) and Early Intervention in Psychosis (EIP) team in the south of England, UK. All referrals over a 4 year 9 month period (March 2014 to December 2018) were included.

\section{Procedure}

Psychologists and psychological therapists work as integrated members of the CMHT and EIP teams, and support engagement through working closely with care co-ordinators and arranging flexible initial contact where needed. All referrals are entered onto a Microsoft access database, which includes demographic information, therapy referred for, waiting time and reason for discharge from therapy. Routinely collected data from medical records was also analysed. As the evaluation used existing routine data anonymously, the project was approved by the local NHS research and service evaluation team as a service evaluation, who confirmed that NHS ethical approval was not required. Those that were referred multiple times during the time frame were only entered into the analysis once using their first referral.

CBT included High Intensity (HI) therapy with a psychologist or CBT therapist, of at least 16 sessions, in line with NICE guidance (NICE, 2014). All staff had specific training in CBT for psychosis as part of their CBT diploma or doctorate training and most had attended 
post-qualification training on CBT for psychosis at the Oxford Cognitive Therapy Centre. All therapists were accredited or accreditable by the British Association of Behavioural and Cognitive Psychotherapy and received regular one-to-one or peer group supervision.

Low Intensity (LI) therapy was also available with a social worker, nurse or occupational therapist who had experience working clinically with people with psychosis as well as training in an introduction to CBT and symptom specific psychosis interventions such as worry and sleep, in line with the cognitive model of the maintenance of persecutory delusions (Freeman, 2016). Service guidelines recommended approximately eight sessions for LI CBT.

Therapy was usually conducted in the service in an outpatient hospital, although for some participants therapy was conducted in their home or other community settings.

Participants had to have been offered an appointment to start therapy; those who disengaged from the service or asked to be removed from the waiting list whilst waiting were not included in the analysis. Those who were discharged for reasons other than disengaged or therapy completed (for example moved out of area or where therapy was not indicated at assessment) were also excluded from the analysis. Thus disengagement here is defined as being offered an appointment having been accepted for therapy (following assessment or written referral and discussion with team member), and not attending the initial appointments offered, or starting therapy and then not attending further appointments offered by the therapist. For all patients that did not initially attend or start attending, a number of contacts were attempted or further appointments offered prior to discharging for disengagement. Alternatively some were discharged as disengaged due to telling the therapist or another member of staff that they did not wish to continue, or when the patient and therapist agreed to end therapy due to poor attendance. 


\section{Statistical Analysis}

The only missing data for demographic and clinical information from medical records was for ethnicity where five individuals declined to provide this information for their medical records. For the HoNOS, ten individuals did not have all questions answered: six had a single item missing and one had four items missing. For individual missing items of the HoNOS the mean for the sample was used. To ensure statistical power given sample size all categorical variables were converted to two levels. For example, ethnicity (based on self-reported categories from British census on medical records) was categorised into White British vs. Black and Minority Ethnic (BME). The HoNOS total and individual items were analysed. For individual HoNOS items the categories were pooled into two groups (due to low sample size), based on the median response, for example "No problem or minor problem" to "Mild to severe problem.”

Chi Square analyses were used for all categorical variables. No cells had an expected size of less than five for any of the analyses suggesting it was possible to conduct the analysis though there may have been low statistical power given the sample size. A Multivariate Analysis of Variance (MANOVA) was conducted for continuous variables. Data was analysed using Statistics Package for the Social Sciences version 25.

\section{Results}

\section{Participant Characteristics}

A sample of 103 patients with a diagnosis of psychosis was included in the evaluation. Table 1 displays the demographic and clinical characteristics of the overall sample. The sample was $61.2 \%(n=63)$ male and $74.7 \%(n=77)$ of white British ethnicity with a mean age of 37.6 years. Fifty-two per cent $(n=53)$ of referrals were EIP cases. In terms of clinical diagnoses, $25.2 \%(n=26)$ had a diagnosis of schizophrenia, $22.3 \%(n=23)$ first episode psychosis, $8.7 \%$ 
$(n=9)$ drug-induced psychosis, 9.7\% (n=10) schizoaffective disorder, $7.8 \%(n=8)$ delusional disorder, $4.9 \%(n=5)$ bipolar disorder with psychotic symptoms / psychotic mania, $6.8 \%$ $(n=7)$ depression with psychotic symptoms, $8.7 \%(n=9)$ psychosis not otherwise specified, $3.9 \%(n=4)$ acute psychotic episode or acute transient psychotic episode, $1 \%(n=1)$ post-natal psychosis, and 1\% $(\mathrm{n}=1)$ recurring brief psychosis unspecified.

Due to the small sample size for individual diagnoses, it was not possible to examine disengagement rates by diagnosis, though descriptive statistics are shown in table 1 .

\section{**Insert Table 1 about here**}

\section{Factors associated with Disengagement}

Overall, $57.3 \%(n=59)$ of people completed therapy, and $42.7 \%(n=44)$ disengaged. For LICBT completers the mean number of sessions attended was 9.5, for HI-CBT this was 16.5 . For those who disengaged from LI-CBT, the mean number of sessions attended was 1.5 with $20 \%(n=2)$ not attending any appointments. For those who disengaged from HI-CBT the mean number of sessions attended was 3.2 with $29.4 \% \quad(n=10)$ not attending any appointments.

For those who disengaged, the mean number of sessions cancelled or not attended was 3.3. In terms of how the final reason to discharge as disengaged was taken: $38.6 \%(n=17)$ did not make contact when requested or could not be contacted; in these cases a mean of three contacts (text, email, phone or letter) were made to seek re-engagement. In $22.7 \%(n=10)$ of cases the therapist discussed concerns about engagement with the patients' psychiatrist or care coordinator and came to a joint decision to discharge. In $11.4 \%(n=5)$ the patient told another member of staff that they did not wish to continue CBT. In $11.4 \%(n=5)$ the patient told the therapist they wanted to stop. Finally, in $15.9 \%(n=5)$ of cases the therapist and 
patient agreed together to discharge due to the number of sessions missed, homework not being completed or agreeing that it was not the right time to engage in therapy.

The results for differences in disengagement rates by categorical variables identified from clinical notes are shown in table 1. Table 2 shows differences in disengagement by categorical variables from individual HoNOS items. Table 3 shows the differences in disengagement based on continuous variables.

\section{**Insert Tables 2 and 3 about here $* *$}

None of the clinical variables (except HoNOS scores - see below) predicted disengagement, and there was no impact of demographic variables of ethnicity or gender. Risk of disengagement was significantly higher for those of younger age ( $M=40.5$ years for completers and 33.8 years for those disengaged): $F=6.89$, partial $\eta^{2}=.064, p=<.05$.

Total HoNOS score did predict disengagement, with higher scores associated with greater likelihood of disengagement $(M=14.5$ versus 11.7$): F=4.22$, partial $\eta^{2}=.040, p<.05$. There was a non-significant trend for shorter waiting times in those who disengaged $(M=14.1$ vs. 18.3 weeks).

Individual HoNOS items were also significant with higher disengagement more likely for those with more severe symptoms on overactive, aggressive, disruptive or agitated behaviour $(54.9 \%$ vs. $30.8 \%): \chi^{2}=6.13, p<.01$; problem drinking or drug taking $(61.1 \%$ vs. $32.8 \%): \chi^{2}=7.65, p<.01 ;$ depressed $\operatorname{mood}(56 \%$ vs. $30.2 \%): \chi^{2}=7.0, p<.01$; and those with greater problems with occupation and activities (53.3\% vs. $34.5 \%): \chi^{2=3.68, p<.05}$. 


\section{Discussion}

This service evaluation aimed to determine predictors of disengagement from CBT for people with psychosis. This builds on the modest literature examining disengagement from multidisciplinary services for this group, and adds to the literature for CBT specifically in routine NHS settings. Just over half of all referrals completed therapy. This is a higher rate of disengagement than has been found for Early Intervention Services in general (Doyle et al., 2014), possibly because of the motivation required to engage in CBT compared to other interventions such as medical reviews. Disengagement rates were also higher than for an IAPT Serious Mental Illness (SMI) service evaluation (Jolley et al., 2015), this may be because the inclusion criteria differed in the current service. The high proportion of people who failed to attend any sessions suggests that facilitating initial commitment to CBT may be a significant issue.

Most of the demographic and clinical variables identified from the clinical notes were not associated with disengagement. There were no differences based on gender or ethnicity, consistent with some (Doyle et al., 2014) but not all previous studies (Kreyenbuhl et al., 2009). Younger people were more likely to drop out, again consistent with some (Kreyenbuhl et al., 2009) but not other past studies (Doyle et al., 2014). It is possible that age is confounded with other variables which may increase risk of disengagement such as substance misuse. A larger sample size would be required to conduct a multiple regression to see if this is the case.

There was a non-significant trend for those who disengaged to have waited less time for therapy. This is perhaps in the opposite direction to what might be expected. Previous research has shown that adolescents are more likely to decline family therapy if they face longer waiting times (Westin, Barksdale \& Stephan, 2014). Another study found that those attending outpatient psychological therapy were more likely not to attend the initial 
appointment if there was a longer delay, though there was no impact on engagement once therapy had started (Reitzel et al (2006). Further research is needed to explore the impact of waiting times on engagement in CBT for psychosis.

Total HoNOS and four specific items also predicted disengagement: overactive, aggressive, disruptive or agitated behaviour; problem drinking or drug taking, problems with depressed mood; and problems with occupation and activities. The link with disturbed behaviour is a new finding and requires replication before any firm conclusions can be drawn. The systematic review completed by Doyle et al. (2014) found that substance misuse consistently predicted increased risk of disengagement from EIP services (Doyle et al., 2014). The current findings suggest that this is also the case for CBT specifically, but for current rather than historical misuse. The link between disengagement and depressed mood is consistent with previous work suggesting that symptoms of depression may be related to psychosis symptom severity and risk of relapse, and should be therefore be targeted in therapy (Hartley, Barrowclough, \& Haddock, 2013). The current findings add to this and suggest that addressing depression early on may increase engagement in therapy.

Interestingly, while global symptom severity / complexity was associated with disengagement, the presence of psychotic symptoms at the point of referral and severity of hallucinations and delusions were not. These negative results combined with the finding of greater disengagement in those with problems with occupation and activities, suggest that it is not the symptoms of psychosis, such as delusions, which hinder engagement with CBT, but rather linked behavioural, emotional and possibly social and occupational factors.

The mean number of sessions of 9.5 for LI-CBT was similar to the mean of 9 in a meta-analysis of LI-CBT for psychosis (Hazell, Hayward, Cavanagh, \& Strauss, 2016). The finding that LI CBT was not linked to higher levels of disengagement supports the utility of non-psychologist therapists delivering focused, manualised interventions targeting specific 
maintenance factors for distressing psychosis, in line with cognitive models of persecutory delusions (Freeman, 2016). However, it is also likely that those referred for HI-CBT had more severe difficulties which may have impacted on engagement. Greater use of such LI interventions where this is indicated by an individual's presentation may help increase access to CBT-based interventions for psychosis.

\section{Clinical implications}

If it is not psychosis per se but secondary or concurrent factors that increase the risk of disengagement from CBT, these variables could be routinely screened at the point of referral / assessment to identify those at greater risk of disengaging, and then targeted prior to therapy or early in therapy to increase likelihood of engagement. This might be most efficiently done with the person's care co-ordinator or support worker whilst waiting for therapy to commence.

Given the impact of disturbed behaviour and depression, assertive engagement may be valuable for people struggling with chaotic lifestyles and low mood, particularly for younger people accessing services. This might include practical prompts such as phone calls and liaising closely with the person's care co-ordinator (Lal \& Malla, 2015). CBT has shown promise for the negative symptoms of psychosis (Staring, ter Huurne, \& van der Gaag, 2013), which may include low mood, and so prioritising this early in CBT may be beneficial both in terms of improving symptoms of depression, and increasing likelihood of completing therapy.

Motivational work is an important component of CBT adapted for those with psychosis and substance use problems (Baker et al., 2006), and is likely to be essential in facilitating inital engagement for those struggling with substance misuse alongside their psychosis. More broadly, this may also include reconsidering models of service delivery to 
improve engagement and access, for example being flexible about timing of contacts and offering online or telephone interventions. Mulligan et al. (2014) found comparable client rated therapeutic alliance for telephone and face-to-face CBT for psychosis, suggesting that alternative models may be welcomed, particularly by young people with psychosis, identified here as being more likely to disengage.

The association between substance misuse and disengagement is probably the most consistent finding across the literature to date. This highlights the importance of assessing for substance use prior to CBT referrals, and focusing on engagement of the person and family members as per the NICE (2014) guidelines.

The impact of problems of occupation and activities requires further investigation. Given the outcomes for occupational therapy with people with psychosis (Lutgens, Gariepy, \& Malla, 2017), and the NICE guideline (2014) recommending that vocational / occupational support be provided routinely, this intervention might have the additional benefit of improving engagement if offered prior to CBT.

\section{Limitations and Future Research}

While this evaluation has the advantage of being based in routine clinical practice, it is limited by a relatively small sample size and being retrospective. A prospective research study collecting self-reported clinical data and using standardised measures of symptoms severity would be more effective in determining predictors of dropout. It is important to note that some of the patients included here had primary affective disorder diagnoses; the content of therapy sessions may have therefore focused on mood as well as psychotic symptoms. Additionally, the evaluation adopted a pragmatic definition of disengagement; the lack of a well operationalised and broadly agreed definition of disengagement remains problematic for research in this area (Doyle et al., 2014), though the difference between the number of 
sessions attended between those who completed and those who disengaged suggests a clear distinction between the two groups.

Though all staff were suitably qualified and received regular supervision, there was no monitoring of therapy adherence using standardised measures as has been done in CBT trials (Garety et al., 2008).

A number of statistical analyses were conducted so there is a risk of a type I error. Due to small sample size it is also possible that some of the non-significant results are due to low statistical power. A larger sample would have permitted the use of multiple regression to determine which results remain significant when all are added to the analysis, for example whether younger age remains predictive once substance use is added to the analysis.

The current evaluation drew on the therapy, demographic and clinical variables routinely collected in the services, but these did not include all implicated in the literature. Specifically, we did not measure family involvement, duration of untreated psychosis (Doyle et al., 2014), negative symptoms and educational attainment (Fanning et al., 2012). Primary care psychological services (IAPT) now routinely collect extensive standardised data which can be used to examine engagement and outcomes in some detail. Outside research settings, secondary care services may be relatively poor at collecting and reviewing comparable data.

In order to understand and address disengagement from psychological therapies for people with psychosis, a clear consensus on the definition of disengagement is needed as a priority. A prospective study using standardised measures and variables beyond those routinely collected in secondary care settings is required, including a specific measure of engagement in services for people with psychosis (O'Brien, White, Fahmy, \& Singh, 2009). 


\section{Conclusion}

CBT is recommended for people with psychosis, yet disengagement rates are high and many people do not attend initial appointments. A better understanding of the barriers to engagement with psychological therapies is needed in order to improve outcomes. This evaluation adds to the modest literature to date, and suggests that overactive, aggressive, disruptive or agitated behaviour, substance misuse, depressed mood, problems with occupation and activities, younger age and higher overall severity / complexity of problems, predict disengagement. Importantly, severity of psychotic symptoms may not hinder access to CBT. Linked behavioural, emotional and possibly social and occupational factors might be targeted prior to or early in therapy to engage people in CBT for psychosis. 


\section{References}

Baker, A., Bucci, S., Lewin, T. J., Kay-Lambkin, F., Constable, P. M., \& Carr, V. J. (2006). Cognitive-behavioural therapy for substance use disorders in people with psychotic disorders - Randomised controlled trial. British Journal of Psychiatry, 188, 439-448. doi: 10.1192/bjp.188.5.439

Doyle, R., Turner, N., Fanning, F., Brennan, D., Renwick, L., Lawlor, E., \& Clarke, M. (2014). First-episode psychosis and disengagement from treatment: a systematic review. Psychiatric Services, 65(5), 603-611. doi: 10.1176/appi.ps.201200570

Fanning, F., Foley, S., Lawlor, E., McWilliams, S., Jackson, D., Renwick, L., . . Trimble, T. (2012). Group cognitive behavioural therapy for first episode psychosis: who's referred, who attends and who completes it? Early Intervention in Psychiatry, 6(4), 432-441. doi: 10.1111/j.1751-7893.2011.00333.x

Freeman, D. (2016). Persecutory delusions: a cognitive perspective on understanding and treatment. The Lancet Psychiatry, 3(7), 685-692. doi: 10.1016/S2215-0366(16)000663

Garety, P. A., Fowler, D. G., Freeman, D., Bebbington, P., Dunn, G., \& Kuipers, E. (2008). Cognitive-behavioural therapy and family intervention for relapse prevention and symptom reduction in psychosis: randomised controlled trial. The British Journal of Psychiatry, 192(6), 412-423. doi: 10.1192/bjp.bp.107.043570

Hartley, S., Barrowclough, C., \& Haddock, G. (2013). Anxiety and depression in psychosis: a systematic review of associations with positive psychotic symptoms. Acta Psychiatrica Scandinavica, 128(5), 327-346. doi: 10.1111/acps.12080 
Hazell, C. M., Hayward, M., Cavanagh, K., \& Strauss, C. (2016). A systematic review and meta-analysis of low intensity CBT for psychosis. Clinical psychology review, 45, 183-192. doi: 10.1016/j.cpr.2016.03.004

Jolley, S., Garety, P., Peters, E., Fornells-Ambrojo, M., Onwumere, J., Harris, V., . . Johns, L. (2015). Opportunities and challenges in Improving Access to Psychological Therapies for people with Severe Mental Illness (IAPT-SMI): evaluating the first operational year of the South London and Maudsley (SLaM) demonstration site for psychosis. Behaviour research and therapy, 64, 24-30. doi: 10.1016/j.brat.2014.11.006

Lal, S., \& Malla, A. (2015). Service engagement in first-episode psychosis: current issues and future directions. The Canadian Journal of Psychiatry, 60(8), 341-345. doi: $10.1177 / 070674371506000802$

Lutgens, D., Gariepy, G., \& Malla, A. (2017). Psychological and psychosocial interventions for negative symptoms in psychosis: systematic review and meta-analysis. The British Journal of Psychiatry, 210(5), 324-332. doi: 10.1192/bjp.bp.116.197103

Mulligan, J., Haddock, G., Hartley, S., Davies, J., Sharp, T., Kelly, J., . . Price, J. (2014). An exploration of the therapeutic alliance within a telephone-based cognitive behaviour therapy for individuals with experience of psychosis. Psychology and Psychotherapy: Theory, Research and Practice, 87(4), 393-410. doi: 10.1111/papt.12018

NICE. (2014). Psychosis and schizophrenia in adults: prevention and management. Retrieved from London:

O'Brien, A., White, S., Fahmy, R., \& Singh, S. P. (2009). The development and validation of the SOLES, a new scale measuring engagement with mental health services in people with psychosis. Journal of Mental Health, 18(6), 510-522. doi: $10.3109 / 09638230902968225$ 
Reitzel, L. R., Stellrecht, N. E., Gordon, K. H., Lima, E. N., Wingate, L. R., Brown, J. S., . . Joiner Jr, T. E. (2006). Does time between application and case assignment predict therapy attendance or premature termination in outpatients? Psychological Services, 3(1), 51. doi: 10.1037/1541-1559.3.1.51

Statistics Package for the Social Sciences (2013). IBM SPSS Statistics for Windows, Version 22.0. Armonk, NY: IBM Corp.

Staring, A. B., ter Huurne, M.-A. B., \& van der Gaag, M. (2013). Cognitive Behavioral Therapy for negative symptoms (CBT-n) in psychotic disorders: a pilot study. Journal of behavior therapy and experimental psychiatry, 44(3), 300-306. doi: 10.1016/j.jbtep.2013.01.004

Westin, A. M., Barksdale, C. L., \& Stephan, S. H. (2014). The effect of waiting time on youth engagement to evidence based treatments. Community mental health journal, 50(2), 221-228. Wing, J., Beevor, A., Curtis, R., Park, S., Hadden, J., \& Burns, A. (1998). Health of the Nation Outcome Scales (HoNOS): research and development. The British Journal of Psychiatry, 172(1), 11-18. doi: https://doi.org/10.1192/bjp.172.1.11 


\section{Table 1}

Differences in disengagement rates by categorical variables identified from routine clinical notes

\begin{tabular}{|c|c|c|c|c|}
\hline \multicolumn{2}{|c|}{ Categorical variables from clinical notes } & $\begin{array}{l}\text { Proportion of } \\
\text { sample }\end{array}$ & $\begin{array}{l}\text { Disengagement rates } \\
\text { (Overall: } 42.7 \%, n=44)\end{array}$ & $\begin{array}{l}\text { Chi-square and } \\
\text { significance }\end{array}$ \\
\hline \multirow[t]{2}{*}{ Gender } & Male & $61.2 \%(n=63)$ & $41.3 \%(n=26)$ & $\chi^{2}=.14, N S$ \\
\hline & Female & $38.8 \%(n=40)$ & $45 \%(n=18)$ & \\
\hline \multirow[t]{2}{*}{ EIP / CMHT } & EIP & $51.5 \%(n=53)$ & $48 \%(n=24)$ & $\chi^{2}=.1 .11, N S$ \\
\hline & CMHT & $48.5 \%(n=50)$ & $37.7 \%(n=20)$ & \\
\hline \multirow[t]{2}{*}{ CBT intensity } & High intensity & $79.6 \%(n=82))$ & $41.5 \%(n=34)$ & $\chi^{2}=.26, N S$ \\
\hline & Low intensity & $20.4 \%(n=21)$ & $47.6 \%(n=10)$ & \\
\hline Historical substance & Present & $67.0 \%(n=69)$ & $46.4 \%(n=32)$ & $\chi^{2}=1.14, N S$ \\
\hline misuse & None & $33.0 \%(n=34)$ & $35.3 \%(n=12)$ & \\
\hline \multirow[t]{3}{*}{ Ethnicity } & White British & $74.7 \%(n=77)$ & $42.9 \%(n=33)$ & $\chi^{2}=1.12, N S$ \\
\hline & BME & $19.4 \%(n=20)$ & $50 \%(n=10)$ & \\
\hline & Not reported & $5.8 \%(n=6)$ & & \\
\hline Diagnosis & Schizophrenia & $25.2 \%(n=26)$ & $26.9 \%(n=7)$ & Not calculated \\
\hline
\end{tabular}




\begin{tabular}{|c|c|c|c|c|}
\hline & First episode psychosis & $22.3 \%(n=23)$ & $65.2 \%(n=15)$ & \\
\hline & Drug-induced psychosis & $8.7 \%(n=9)$ & $77.8 \%(n=7)$ & due to sample \\
\hline & Schizoaffective disorder & $9.7 \%(n=10)$ & $60 \%(n=6)$ & size \\
\hline & Delusional disorder & $7.8 \%(n=8)$ & $25 \%(n=2)$ & \\
\hline & Bipolar / mania with psychosis & $4.9 \%(n=5)$ & $0 \%(n=0)$ & \\
\hline & Depression with psychotic symptoms & $6.8 \%(n=7)$ & $14.3 \%(n=1)$ & \\
\hline & Psychosis not otherwise specified & $8.7 \%(n=9)$ & $44.4 \%(n=4)$ & \\
\hline & Acute psychotic episode or acute & $3.9 \%(n=4)$ & $50 \%(n=2)$ & \\
\hline & transient psychotic episode & & & \\
\hline & Post-natal psychosis & $1 \%(n=1)$ & $0 \%(n=0)$ & \\
\hline & Recurring brief psychosis unspecified & $1 \%(n=1)$ & $0 \%(n=0)$ & \\
\hline Co-Morbid & Present & $37.9 \%(n=39)$ & $51.3 \%(n=20)$ & $\chi^{2}=1.88, N S$ \\
\hline Diagnosis & None & $62.1 \%(n=64)$ & $47.5 \%(n=24)$ & \\
\hline Acute care in past & Yes & $46.6 \%(n=48)$ & $43.8 \%(n=21)$ & $\chi^{2}=.04, N S$ \\
\hline year & No & $53.4 \%(n=55)$ & $41.8 \%(n=23)$ & \\
\hline Positive symptoms & Yes & $66.0 \%(n=68)$ & $38.2 \%(n=26)$ & $\chi^{2}=1.64, N S$ \\
\hline
\end{tabular}




$\begin{array}{lllll}\text { at referral } & \text { No } & 33.9 \%(n=35) & 51.4 \%(n=18) \\ \text { History of } & \text { Yes } & 43.7 \%(n=45) & 44.4 \%(n=20) & \chi 2=0.10, N S \\ \text { aggression } & \text { No } & 56.3 \%(n=58) & 4143 \%(n=24) & \\ \text { History of self-harm } & \text { Yes } & 44.6 \%(\mathrm{n}=46) & 50 \%(n=23) & \chi 2=1.80, N S \\ \text { or suicide attempts } & \text { No } & 55.3 \%(\mathrm{n}=57) & 36.81 \%(n=21) & \end{array}$

$N S=$ Non-Significant 


\section{Table 2}

Differences in disengagement rates by HoNOS categorical variables

\begin{tabular}{|c|c|c|c|c|}
\hline \multicolumn{2}{|l|}{ HoNOS categorical variables } & \multirow{2}{*}{$\begin{array}{l}\text { Proportion of } \\
\text { sample }\end{array}$} & \multirow{2}{*}{$\begin{array}{l}\text { Disengagement rates } \\
\text { Overall }=42.7 \%(n=44)\end{array}$} & \multirow{2}{*}{$\begin{array}{l}\text { Chi-Square and } \\
\text { significance }\end{array}$} \\
\hline & & & & \\
\hline 1. Overactive, aggressive, disruptive or & No problem & $50.5 \%(n=52)$ & $30.8 \%(n=16)$ & $\chi^{2}=6.13, p<.01$, \\
\hline \multirow[t]{2}{*}{ agitated behaviour } & & & & one-tailed. \\
\hline & Minor to severe problem & $49.5 \%(n=12)$ & $54.9 \%(n=28)$ & \\
\hline \multirow[t]{2}{*}{ 2. Non-accidental self-Injury } & No problem & $76.7 \%(n=79)$ & $41.8 \%(n=33)$ & $\chi^{2}=.12, N S$ \\
\hline & Minor to severe problem & $23.3 \%(n=24)$ & $45.8 \%(n=11)$ & \\
\hline \multirow[t]{3}{*}{ 3. Problem drinking or drug taking } & No problem & $65.1 \%(n=67)$ & $32.8 \%(n=22)$ & $\chi^{2}=7.65, p<.01$, \\
\hline & & & & one-tailed. \\
\hline & Minor to severe problem & $34.9 \%(n=36)$ & $61.1 \%(n=22)$ & \\
\hline \multirow[t]{2}{*}{ 4. Cognitive Problems } & No problem & $68.9 \%(n=71)$ & $45.8 \%(n=32)$ & $\chi^{2}=.52, N S$ \\
\hline & Minor to severe problem & $31.1 \%(n=32)$ & $37.5 \%(n=12)$ & \\
\hline \multirow[t]{2}{*}{ 5. Physical illness or Disability } & No problem & $62.1 \%(n=64)$ & $46.9 \%(n=30)$ & $\chi^{2}=1.19, N S$ \\
\hline & Minor to severe problem & $37.8 \%(n=39)$ & $35.9 \%(n=14)$ & \\
\hline
\end{tabular}


6. Problems associated with

hallucinations and delusions

7. Problems with depressed mood

8. Other mental and behavioural

problems

9. Problems with relationships

10. Problems with activities of daily

living

11. Problems with living conditions

12. Problems with occupation and activities
No to mild problem

$62.1 \%(n=64)$

$45.3 \%(n=29)$

$\chi 2=.47, N S$

Moderate to severe problem

$37.9 \%(n=39)$

$38.5 \%(n=15)$

No to minor problem

$51.5 \%(n=53)$

$30.2 \%(n=16)$

$\chi 2=7.0, p<.01$, one-

tailed

Mild to severe problem

No to minor problem

$48.5 \%(n=50) \quad 56 \%(n=28)$

$42.7 \%(n=44) \quad 36.4 \%(n=16)$

$\chi 2=127, N S$

Mild to severe problem

$57.3 \%(n=59)$

$47.5 \%(n=28)$

No to minor problem

$55.3 \%(n=57)$

$36.8 \%(n=21)$

$\chi 2=1.8, N S$

Mild to severe problem

$44.7 \%(n=46)$

$50 \%(n=23)$

No to minor problem

$57.3 \%(n=59)$

$39 \%(n=23)$

$\chi 2=.79, N S$

Mild to severe problem

$42.7 \%(n=44)$

$47.7 \%(n=21)$

No problem

$66.0 \%(n=68)$

$39.7 \%(n=27)$

$\chi 2=.74, N S$

Minor to severe problem

$33.9 \%(n=35)$

$48.6 \%(n=17)$

No to minor problem

$56.3 \%(n=58)$

$34.5 \%(n=20)$ $\chi 2=3.68, p<.05$,

one-tailed 


\section{Table 3}

Differences in disengagement rates by continuous variables identified from routine clinical notes

\begin{tabular}{llll}
\hline Continuous variables & Whole sample & Means for completers & MANOVA and \\
from clinical notes & & and disengagers & significance \\
\hline Age & Range: $18-66$ & Completers $=40.5$ & $F=6.89$, partial \\
& $M=37.6$ years & Disengagers $=33.8$ & $\eta^{2}=.064, p<.05$ \\
Waiting time (weeks) & Range: $0-55$, & Completers $=18.3$ & $F=2.8$, partial \\
& $M=16.5$ & Disengagers $=14.1$ & $\eta^{2}=.027, p=.09$ \\
& Range: $0-35$, & Completers $=11.7$ & $F=4.22$, partial \\
& $M=12.9$ & Disengagers $=14.5$ & $\eta^{2}=.040, p<.05$ \\
\hline
\end{tabular}

$N S=$ non-significant 